\title{
Topological classification of dynamical phase transitions
}

\author{
Szabolcs Vajna and Balázs Dóra \\ Department of Physics and BME-MTA Exotic Quantum Phases Research Group, Budapest University of Technology and Economics, \\ 1521 Budapest, Hungary
}

(Received 1 October 2014; revised manuscript received 20 March 2015; published 17 April 2015)

\begin{abstract}
We study the nonequilibrium time evolution of a variety of one-dimensional (1D) and two-dimensional (2D) systems (including SSH model, Kitaev-chain, Haldane model, $p+i p$ superconductor, etc.) following a sudden quench. We prove analytically that topology-changing quenches are always followed by nonanalytical temporal behavior of return rates (logarithm of the Loschmidt echo), referred to as dynamical phase transitions (DPTs) in the literature. Similarly to edge states in topological insulators, DPTs can be classified as being topologically protected or not. In 1D systems the number of topologically protected nonequilibrium time scales are determined by the difference between the initial and final winding numbers, while in 2D systems no such relation exists for the Chern numbers. The singularities of dynamical free energy in the $2 \mathrm{D}$ case are qualitatively different from those of the 1D case; the cusps appear only in the first time derivative.
\end{abstract}

DOI: 10.1103/PhysRevB.91.155127

PACS number(s): 64.70.Tg, 05.30.Rt, 05.70.Ln

Topology [1,2] and nonequilibrium dynamics [3,4] are two vividly investigated fields of physics, with no strong bonds between them. The leading role played by topology in condensed matter has only been realized recently with the discovery of topological insulators, the descendants of quantum Hall states. Some of their correlation functions are universal and are not influenced by the microscopic details of the system, but are rather determined by the underlying topology. The analysis of nonequilibrium states, on the other hand, have emerged recently in a different field: in cold atomic systems. With the unprecedented control of preparing initial states and governing the time evolution, a number of interesting phenomena has been observed such as the Kibble Zurek scaling [5], the lack of thermalization in integrable systems [6], etc. In this paper, we connect these two, seemingly unrelated fields and show that topology can be used as an organizing principle to classify out-of-equilibrium systems.

The most popular setups for nonequilibrium dynamics are quench experiments in which the quantum system initially sits in the ground state of a given Hamiltonian, but its time evolution is governed by another Hamiltonian. The quench protocol can conveniently be characterized by the dynamical partition function with no reference to any particular observables, defined as

$$
Z(z)=\left\langle\psi\left|e^{-H z}\right| \psi\right\rangle
$$

For positive real values of $z$ this gives the partition function of a field theory with boundaries $|\psi\rangle$ separated by $z$ [7]. For our purposes, we use $z=i t$ with $t$ real, which then gives the Loschmidt amplitude, that is, the overlap of the time-evolved state with the initial state $G(t)=Z(i t)$. It characterizes time evolution and the stationary state after a long waiting time [8], and also yields the characteristic function of work [9], which is accessible experimentally [10]. As the time evolution operator is highly nonlocal, it allows $Z(z)$ to be susceptible to the topological properties of the underlying system. Similarly to the equilibrium situation, the dynamical free energy is defined as the logarithm per unit volume $f(t)=-1 / N^{d} \ln G(t)$. In the thermodynamic limit it can be a nonanalytical function of time, which was dubbed DPTs [11]. Although we are mostly interested in the dynamical partition function for imaginary arguments, following Fisher's method of studying phase transitions, its structures are revealed by analyzing the function on the whole complex plane. Fisher's method also supports the analogy between phase transitions and DPTs. His idea was to study the zeros of the partition function, because they are responsible for nonanalyticities in the free energy [12].

Former studies of DPTs mainly focused on spin systems $[8,11,13-21]$, and DPTs were shown to appear not only in free, but in interacting systems as well. However, in the latter case only specific models were addressed (e.g., XXZ Heisenberg chain or Hubbard model) mostly numerically, therefore the generality and applicability of these results to other models are not clear. The topological properties are also less understood in interacting systems, which are fundamental components of our work. In contrast, we give a comprehensive description of a family of noninteracting models which covers a large variety of topological and superconducting systems, and we demonstrate the prominent role of topology in quench dynamics.

We consider two-band translational invariant insulators and Bogoliubov-de Gennes superconductors in one and two dimensions. Such systems are widely studied in the literature, and some of them had already been realized experimentally, either in cold atomic or in condensed matter systems [22-25]. The Hamiltonian for these systems can be parametrized by a vector $\mathbf{d}_{\mathbf{k}}$,

$$
H=\sum_{\mathbf{k}} \mathbf{c}_{\mathbf{k}}^{+} h_{\mathbf{k}} \mathbf{c}_{\mathbf{k}}, \quad h_{\mathbf{k}}=\mathbf{d}_{\mathbf{k}} \cdot \sigma
$$

where $\mathbf{c}_{\mathbf{k}}^{+}=\left(c_{\mathbf{k}, A}^{+}, c_{\mathbf{k}, B}^{+}\right)$for insulators and $\mathbf{c}_{\mathbf{k}}^{+}=\left(c_{\mathbf{k}}^{+}, c_{-\mathbf{k}}\right)$ for superconductors. In the insulating case the internal degrees of freedom $A, B$ refer to pseudospin components, e.g., to different sublattices. A sudden quench protocol can be described by the change in the vector fields characterizing the Hamiltonian: $\mathbf{d}_{\mathbf{k}}(t)=\mathbf{d}_{\mathbf{k}}^{0}$ for $t<0$ and $\mathbf{d}_{\mathbf{k}}(t)=\mathbf{d}_{\mathbf{k}}^{1}$ for $t>0$. The Loschmidt amplitude following this quench can be expressed in a compact form independently of the spatial dimensions:

$$
G(t)=\prod_{\mathbf{k}}\left[\cos \left(\epsilon_{\mathbf{k}}^{1} t\right)+i \hat{\mathbf{d}}_{\mathbf{k}}^{0} \cdot \hat{\mathbf{d}}_{\mathbf{k}}^{1} \sin \left(\epsilon_{\mathbf{k}}^{1} t\right)\right],
$$


but the product is taken for all wave numbers in the Brillouin zone for normal insulators and for half of the Brillouin zone for superconductors. Here, $\hat{\mathbf{d}}_{\mathbf{k}}^{i}$ denotes the unit vector in the direction of $\mathbf{d}_{\mathbf{k}}^{i}$ and $\epsilon_{\mathbf{k}}^{i}=\left|\mathbf{d}_{\mathbf{k}}^{i}\right|$ for insulators and $\epsilon_{\mathbf{k}}^{i}=2\left|\mathbf{d}_{\mathbf{k}}^{i}\right|$ for superconductors. The Fisher zeros, i.e., the solutions of $Z(z)=0$ are

$$
z_{n}(\mathbf{k})=\frac{i \pi}{\epsilon_{\mathbf{k}}^{1}}\left(n+\frac{1}{2}\right)-\frac{1}{\epsilon_{\mathbf{k}}^{1}} \operatorname{arth}\left[\hat{\mathbf{d}}_{\mathbf{k}}^{0} \cdot \hat{\mathbf{d}}_{\mathbf{k}}^{1}\right],
$$

which follow from the product form of the Loschmidt amplitude. The Fisher zeros fill domains of the complex plane, which are indexed by an integer number $n$ and are parametrized with $\mathbf{k}$. In one dimension these domains form lines, while in two dimensions they fill areas. The necessary condition to observe DPTs is having Fisher zeros approaching the imaginary axis, which occurs when $\mathbf{d}_{\mathbf{k}}^{0} \cdot \mathbf{d}_{\mathbf{k}}^{1}=0$, i.e., when the vector $\mathbf{d}_{\mathbf{k}}$ in the final Hamiltonian is perpendicular to the initial one for some $\mathbf{k}$. This geometrical condition connects DPTs with the topology of the initial and the final systems. In the following we will consider the one- and two-dimensional cases separately.

One-dimensional case. Topological insulators in one dimension are characterized by chiral (AIII symmetry class) or chiral and particle-hole symmetry (BDI) [26], which constrain $\mathbf{d}_{k}$ to lie in a $2 \mathrm{D}$ plane. The corresponding topological number is the winding number: the number of times $\mathbf{d}_{k}$ winds around the origin when $k$ sweeps through the Brillouin zone. If, for example, $\mathbf{d}_{k}$ lies in the $x y$ plane, $v=\frac{1}{2 \pi} \int d k\left(\hat{d}_{k}^{x} \partial_{k} \hat{d}_{k}^{y}-\right.$ $\left.\hat{d}_{k}^{y} \partial_{k} \hat{d}_{k}^{x}\right)$.

If the winding number of two vector fields $\mathbf{d}_{k}^{0}$ and $\mathbf{d}_{k}^{1}$ defined on the Brillouin zone $\left(S^{1}\right)$ differ by $\Delta v \in \mathbb{N}$, the image of the scalar product field $\hat{\mathbf{d}}_{k}^{0} \cdot \hat{\mathbf{d}}_{k}^{1}$ covers the interval $[-1,1]$ at least $2 \Delta v$ times.

This means that the Fisher zeros in Eq. (4) sweep through the real axis $2 \Delta v$ times while $k$ goes through the Brillouin zone. Consequently there are at least $2 \Delta v$ points in $k$ space where the vectors are perpendicular [for illustration see Fig. 1(a)],

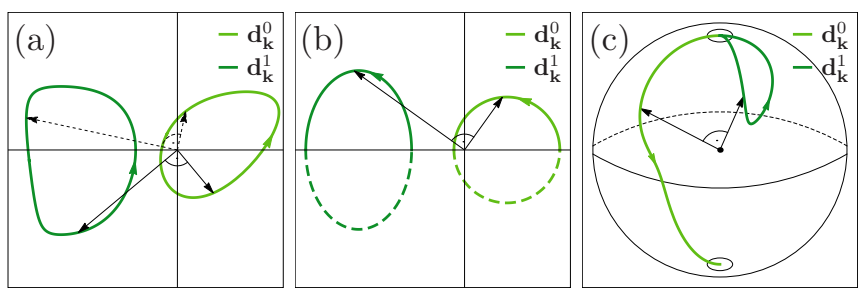

FIG. 1. (Color online) Illustration for the existence of perpendicular vectors if the quench connects domains with different topological numbers. (a) Topological insulators in AIII symmetry class. As $k$ goes through the Brillouin zone $\mathbf{d}_{k}$ draws a closed loop. For any parametrization of these loops there will be at least $2 \Delta v$ wave numbers for which $\mathbf{d}^{0} \perp \mathbf{d}^{1}$ if the winding number of the two vector fields differ by $\Delta v$. (b) Superconductors in the BDI symmetry class. In the $k \in[0, \pi]$ domain (solid line) there will be at least $\Delta v$ perpendicular $\mathbf{d}$ vectors in a quench characterized by $\Delta v$. (c) Superconductors in the D symmetry class. The vectors $\hat{\mathbf{d}}$ are no longer confined to a plane, but the occurrence of perpendicular vectors is still ensured when the topology of $\hat{\mathbf{d}}^{1}$ and $\hat{\mathbf{d}}^{0}$ are different. implying DPTs. Let us suppose that the (ground state) winding number of the initial (final) Hamiltonian is $v_{0}\left(v_{1}\right)$, then the angle of rotation $\phi_{k}^{i}$ for $\mathbf{d}_{k}^{i}$ is a smooth function differing by $2 \pi \nu_{i}$ at $k=-\pi$ and $\pi$ for $i=0,1$. The angle of rotation $\Delta \phi_{k}$ between $\mathbf{d}_{k}^{0}$ and $\mathbf{d}_{k}^{1}$ changes $2 \pi \Delta \nu$, hence $\hat{\mathbf{d}}_{k}^{0} \cdot \hat{\mathbf{d}}_{k}^{1}=\cos \left(\Delta \phi_{k}\right)$ covers the interval $[-1,1]$ at least $2 \Delta v$ times. If the model has further symmetries that connect wave numbers $k$ and $-k$ [e.g., inversion or time reversal symmetry (TRS)], the Fisher lines can be doubly degenerate and there will be only $\Delta v$ nonequilibrium time scales, as it happens, for example, in the SSH model (see later).

Our argument applies also for 1D topological superconductors (e.g., the Kitaev chain and its generalization for higher winding numbers [27]) with a little modification. Now the product is taken only for positive momenta in the Loschmidt amplitude in Eq. (3). The Bogoliubov Hamiltonian is particlehole symmetric (PHS) by construction, implying that the $x$ and $y$ components of $\mathbf{d}_{k}$ are odd and the $z$ component is an even function of the wave number: $d_{-k}^{x}=-d_{k}^{x}, d_{-k}^{y}=-d_{-k}^{y}$, and $d_{-k}^{z}=d_{k}^{z}$. For the degenerate momenta $k=0, \pi$, the vector describing the Hamiltonian points to the $z$ direction: $\mathbf{d}_{0 / \pi}=\left(0,0, d_{0 / \pi}^{z}\right)$. If the system is time reversal invariant as well (BDI symmetry class) $d_{k}^{x} \equiv 0$ and the topological number is the winding number similarly to the previous case. Because of PHS the winding of the angle of $\mathbf{d}_{k}$ is already determined in the $k \in(0, \pi)$ domain. That is, if the winding number is $v$, the angle changes $\pi v$ while $k$ goes through the positive half of the Brillouin zone. Therefore for a quench from $v_{0}$ to $v_{1}$ DPTs will appear with $\Delta v$ topologically protected time scales [Fig. 1(b)]. This result applies for the previous studies in the literature for the transverse field Ising model and for the quantum $X Y$ chain [11,18], which can be mapped to Hamiltonians in the form of (2). The topological protection of DPTs for quenches connecting different phases was also revealed for the transverse field Ising chain $[16,28]$.

If TRS is broken (D symmetry class, [26]) $\mathbf{d}_{k}$ is not confined to a $2 \mathrm{D}$ plane. The $\mathbb{Z}_{2}$ invariant is 0 (topologically trivial) if $\hat{\mathbf{d}}_{0}=\hat{\mathbf{d}}_{\pi}(=(0,0, \pm 1))$ and it is 1 (nontrivial) if $\hat{\mathbf{d}}_{0}=-\hat{\mathbf{d}}_{\pi}$. If the quench connects phases with different topology, e.g., $v_{0}=1$ and $v_{1}=0$, there must be a wave number $k^{*}$ for which $\mathbf{d}_{k^{*}}^{0} \cdot \mathbf{d}_{k^{*}}^{1}=0$ [illustrated in Fig. 1(c)], because $\hat{\mathbf{d}}_{0}^{0} \cdot \hat{\mathbf{d}}_{0}^{1}=-\hat{\mathbf{d}}_{\pi}^{0}$. $\hat{\mathbf{d}}_{\pi}^{1}= \pm 1$, hence $\hat{\mathbf{d}}_{\mathbf{k}}^{0} \cdot \hat{\mathbf{d}}_{\mathbf{k}}^{1}$ covers the interval $[-1,1]$.

So far we have demonstrated that the change in topology under a sudden quench is an eligible condition for DPTs to occur. Note that $\mathbf{d}^{0}$ and $\mathbf{d}^{1}$ can become perpendicular accidentally even if the topological numbers do not differ in the initial and final Hamiltonians [18]. This parallels the appearance of the topologically nonprotected edge or surface states in certain systems, whose existence is not connected to topology but is accidental $[1,29,30]$.

Two dimensions. The topological number is the Chern number $Q$ for two-band topological insulators, which is calculated from the vector $\mathbf{d}_{\mathbf{k}}$ defining the Hamiltonian [31],

$$
Q=\frac{1}{4 \pi} \int_{B Z} d k_{x} d k_{y} \hat{\mathbf{d}}_{\mathbf{k}} \cdot\left(\partial_{k_{x}} \hat{\mathbf{d}}_{\mathbf{k}} \times \partial_{k_{y}} \hat{\mathbf{d}}_{\mathbf{k}}\right),
$$

counting how many times the surface defined by $\hat{\mathbf{d}}_{\mathbf{k}}$ covers the unit sphere. We show that if the quench connects phases with Chern numbers differing in their moduli, DPTs will necessarily 
occur. However, DPTs in two dimensions are qualitatively different from those in one dimension, because of the Fisher zeros form areas instead of lines.

If the Chern numbers of two vector fields $\mathbf{d}_{\mathbf{k}}^{0}$ and $\mathbf{d}_{\mathbf{k}}^{1}$ defined on the Brillouin zone $\left(T^{2}\right)$ differ in the modulus $\left|Q_{1}\right| \neq\left|Q_{0}\right|$, the image of the scalar product field $\hat{\mathbf{d}}^{0} \cdot \hat{\mathbf{d}}^{1}$ is $[-1,1]$.

We prove our statement in four steps.

(a) If $\hat{\mathbf{d}}_{\mathbf{k}}^{0} \cdot \hat{\mathbf{d}}_{\mathbf{k}}^{1}>-1 \Rightarrow Q_{1}=Q_{0}$, because there is a continuous mapping $\mathbf{f}_{\mathbf{k}}(\gamma)$ between $\hat{\mathbf{d}}_{\mathbf{k}}^{0}$ and $\hat{\mathbf{d}}_{\mathbf{k}}^{1}$ such that $\left|\mathbf{f}_{\mathbf{k}}(\gamma)\right|>0$.

$$
\begin{aligned}
\mathbf{f}_{\mathbf{k}}(\gamma) & =(1-\gamma) \hat{\mathbf{d}}_{\mathbf{k}}^{0}+\gamma \hat{\mathbf{d}}_{\mathbf{k}}^{1}, \quad \gamma \in[0,1] \\
\left|\mathbf{f}_{\mathbf{k}}(\gamma)\right|^{2} & =1+2 \gamma(1-\gamma)\left(-1+\hat{\mathbf{d}}_{\mathbf{k}}^{0} \cdot \hat{\mathbf{d}}_{\mathbf{k}}^{1}\right)>0 .
\end{aligned}
$$

The inequality in the second line came from the fact that $\gamma(1-\gamma)<1 / 4$ for $\gamma \in[0,1]$. In other words if the vector fields $\hat{\mathbf{d}}_{\mathbf{k}}^{0}$ and $\hat{\mathbf{d}}_{\mathbf{k}}^{1}$ are nowhere antiparallel, then one can continuously deform one into the other. Under this deformation the Chern number does not change [32].

(b) If $Q_{1} \neq Q_{0} \Rightarrow \hat{\mathbf{d}}_{\mathbf{k}}^{0} \cdot \hat{\mathbf{d}}_{\mathbf{k}}^{1}=-1$ for some $\mathbf{k}$. This comes from reversing (a) and can be proved indirectly.

(c) If $Q_{1} \neq-Q_{0} \Rightarrow \hat{\mathbf{d}}_{\mathbf{k}}^{0} \cdot \hat{\mathbf{d}}_{\mathbf{k}}^{1}=1$ for some (other) $\mathbf{k}$. We trace back this statement to (b) by defining the vector field $\hat{\mathbf{d}}_{\mathbf{k}}^{\prime 1} \equiv-\hat{\mathbf{d}}_{\mathbf{k}}^{1}$, which satisfies $Q_{1}^{\prime} \neq Q_{0}$. Hence $\hat{\mathbf{d}}_{k}^{0} \cdot \hat{\mathbf{d}}_{\mathbf{k}}^{\prime 1}=-1$ for some $\mathbf{k}$ implying (c). Supported by the continuity of $\hat{\mathbf{d}}_{\mathbf{k}}^{i}$ when the topology is well defined, combining (b) and (c) finishes the proof.

This argument cannot be generalized to the $Q_{1}=-Q_{0}$ case; a trivial counterexample is given by the quench $\mathbf{d}_{\mathbf{k}}^{1}=$ $-\mathbf{d}_{\mathbf{k}}^{0}$, where the initial and final Chern numbers are the opposite, but $\mathbf{d}^{0} \cdot \mathbf{d}^{1} \equiv-1$.

The statement ensures that Fisher zeros connect $-\infty$ to $\infty$ if the modulus of the Chern number changes under the quench. Nevertheless, one might find Fisher lines connecting $-\infty$ to $\infty$ also when the modulus of the Chern numbers are the same. A 2D system can be thought of as a collection of $1 \mathrm{D}$ chains. If these $1 \mathrm{D}$ systems can be characterized by winding numbers, it is enough to find a pair of these $1 \mathrm{D}$ systems with differing winding numbers to see DPTs. On the other hand, Fisher zeros can also expand through the imaginary axis accidentally similarly to the $1 \mathrm{D}$ case.

In the superconducting case the product in Eq. (3) is taken for the half Brillouin zone. However, because of PHS one gets exactly the same contribution from the other half of the Brillouin zone, so one can express $G(t)^{2}$ as a product over the whole BZ. From this the existence of DPTs follows for quenches connecting superconducting phases with different moduli of the Chern numbers.

Our results on the mappings $\mathbf{d}_{k}^{0} \cdot \mathbf{d}_{k}^{1}$ are important not only in the context of DPTs, but for the entanglement properties as well. Entanglement is a complementary measure to charaterize the time evolution of wave functions without reference to any observables. The time dependence of the entanglement spectrum has been studied in topological systems following a sudden quench [33,34]. Interestingly, the evolution of the entanglement spectrum shows a qualitative difference for quenches where $\mathbf{d}_{k}^{0} \perp \mathbf{d}_{k}^{1}$ for some $k$, which is the very same condition that appeared in the context of DPTs. Further connections between DPTs and the entanglement spectrum is a subject of future research.
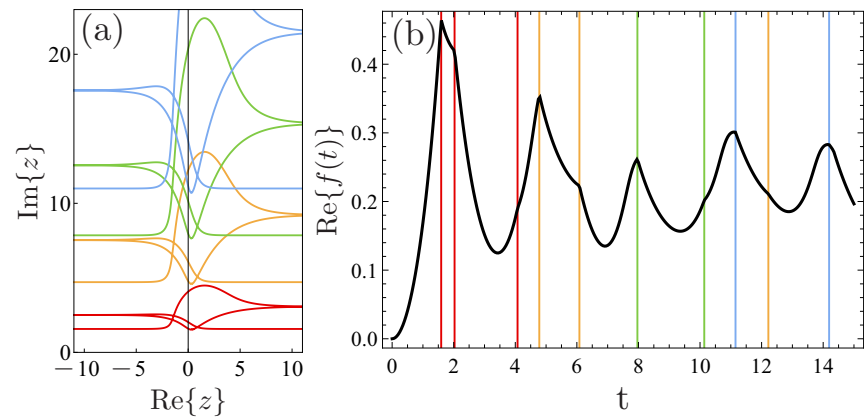

FIG. 2. (Color online) Fisher lines $z_{n}(k)$ (for $n=0 . .3$ ) and DPTs in the generalized SSH model in a quench $v_{0}=1 \rightarrow v_{1}=-2$. (a) The lines of Fisher zeros are doubly degenerate due to the $(k,-k)$ symmetry; they sweep through the real axis $|\Delta v|=3$ times. (b) Dynamical phase transitions appear where the Fisher zeros cross the imaginary axis. The grid lines show the DPTs corresponding to the first four Fisher lines.

Having established our main results, a few examples follow.

Generalized SSH model. The SSH model is a 1D tightbinding chain that was originally introduced to model polyacetylene [35]. It is probably the simplest topological insulator, belonging to the BDI symmetry class [26]. The model is described by $\mathbf{d}_{k}=\left(t_{0}+t_{-1} \cos (k), t_{-1} \sin (k), 0\right)$, where $t_{0}$ and $t_{-1}$ are the staggered hopping amplitudes. The ground state is topologically trivial $(v=0)$ when $t_{0}>t_{-1}$ and is nontrivial if $t_{0}<t_{-1}$. The model can be extended to produce higher winding numbers by introducing longer ranged hopping terms that preserve chiral symmetry. The Hamiltonian in this case is characterized by the vector,

$$
\mathbf{d}_{k}=\left(t_{0}^{\prime}+\sum_{m=1}^{\infty} t_{m}^{\prime} \cos (m k), \sum_{m=1}^{\infty} t_{m}^{\prime \prime} \sin (m k), 0\right),
$$

where $t_{0}^{\prime}=t_{0}, t_{m}^{\prime}=t_{m}+t_{-m}, t_{m}^{\prime \prime}=t_{-m}-t_{m}$, and $t_{m}$ is the real hopping amplitude between sublattices $A$ in unit cell $i$ and $B$ in unit cell $i+m$. We note that in this labeling of the hopping amplitudes $t_{m}$ and $t_{-m}$ are independent and are responsible for the staggered nature of the system. Higher winding numbers can be produced by the proper choice of the hopping amplitudes, for example, the winding number is $|v|=n \geqslant 1$ if $t_{n}^{\prime}$ and $t_{n}^{\prime \prime}$ dominate the other hopping terms. An eligible condition for this is $\left|t_{0}^{\prime}\right|+\sum_{m=1}^{m \neq n}\left|t_{m}^{\prime}\right|+\left|t_{m}^{\prime \prime}\right|<$ $\min \left\{\left|t_{n}^{\prime}\right|,\left|t_{n}^{\prime \prime}\right|\right\}$. Besides the chiral symmetry this model has TRS as well. Therefore in a quench characterized by $\Delta v$, the $2 \Delta v$ topologically protected $(-\infty, \infty)$ sections of the Fisher lines are pairwise degenerate (consider, e.g., that $\left|\mathbf{d}_{k}^{1}\right|=\left|\mathbf{d}_{-k}^{1}\right|$ and $\mathbf{d}_{k}^{0} \cdot \mathbf{d}_{k}^{1}=\mathbf{d}_{-k}^{0} \cdot \mathbf{d}_{-k}^{1}$ ), implying only $\Delta v$ nonequilibrium time scales. The flow of the Fisher lines and the dynamical free energy are shown on Fig. 2 for a quench from a phase with $v_{0}=1$ to $v_{1}=-2$.

The Haldane model is a next-nearest-neighbor hopping model on a honeycomb lattice with artificial magnetic field [36], which can produce topologically nontrivial states. It is characterized by

$$
\mathbf{d}_{\mathbf{k}}=\left(\operatorname{Re}\{f(k)\}, \operatorname{Im}\{f(k)\}, m-g^{\mathrm{asym}}(k, \phi)\right),
$$



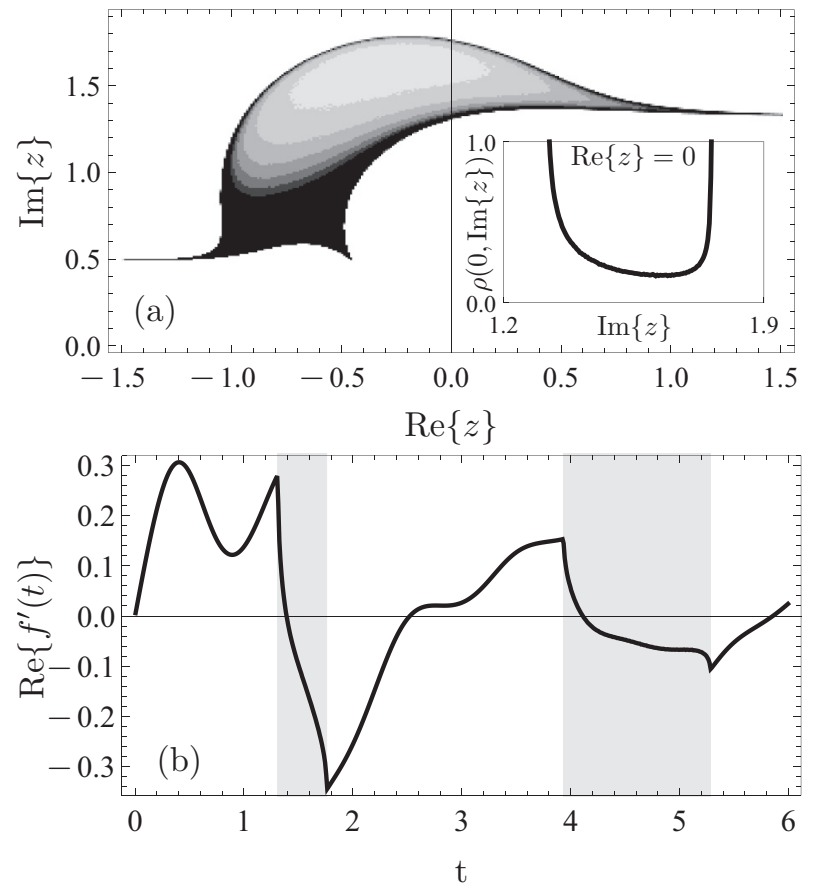

FIG. 3. Quench in the Haldane model from $Q_{0}=0$ to $Q_{1}=1$. (a) Fisher zeros corresponding to $n=0$ in Eq. (4). The normalized density of the Fisher zeros $\rho(\operatorname{Re}\{z\}, \operatorname{Im}\{z\})$ is shown as the darkness of the area. (Inset) The density diverges on the imaginary axis at the boundary of the Fisher area. (b) Cusplike singularities in the first derivative of the dynamical free energy. The shaded areas emphasize the regions where the Fisher zeros cross the time axis.

where $f(k)=\gamma_{1} \sum_{j} e^{-i \mathbf{k} \boldsymbol{\delta}_{j}}$ and vectors $\boldsymbol{\delta}_{j}$ point to the three nearest neighbors. The mass term $m \sigma_{z}$ describes a homogeneous staggered lattice potential. The $-g^{\text {asym }}(\mathbf{k}, \phi) \sigma_{z}$ term comes from a second neighbor hopping considering the staggered magnetic field characterizing the Haldane model. This latter term is necessary to produce nontrivial topology in the model. The Chern number depends on the phase $\phi$ characterizing the magnetic field, on the next-nearest hopping amplitude $\gamma_{2}$, and on the mass term. The Chern number is $Q=$ 0 if $|m|>\left|3 \sqrt{3} \gamma_{2} \sin \phi\right|$, and $Q= \pm 1$ if $|m|<\left|3 \sqrt{3} \gamma_{2} \sin \phi\right|$ with the sign depending on $\phi$ and $\gamma_{2}$.

We have already proved that if the Chern number of the initial and final Hamiltonian differs, the Fisher zeros connect $-\infty$ with $\infty$. However, in contrast to the one-dimensional case, in two dimensions the Fisher zeros fill areas rather than forming lines. Similar behavior occurs for quenches in spin-glass systems [13,37]. In our case the appearance of the Fisher area is not unexpected, since each Fisher domain corresponding to a given $n$ in Eq. (4) is parametrized by two variables $k_{x}$ and $k_{y}$. In contrast to Fisher lines if a Fisher area crosses the imaginary axis, the dynamical free energy looks smooth and its first derivative shows cusps at the boundaries of the Fisher area [Fig. 3(b)]. This is understood by expressing the singular part of the dynamical free energy with the Fisher zeros [11],

$$
f^{s}(t)=-\lim _{N \rightarrow \infty} \frac{1}{N^{d}} \sum_{n, k} \ln \left(1-\frac{i t}{z_{n}(k)}\right) .
$$

If an area with homogeneous density of Fisher zeros crosses the imaginary axis, the second derivative of the dynamical free energy jumps at the boundary of the Fisher area. The size of the jump is proportional to the density of zeros normalized by the system size $(\rho)$ and with the cosine square of the impact angle $(\varphi)$ of the boundary line.

$$
\lim _{\epsilon \rightarrow 0+} f^{\prime \prime}\left(t_{0}+\epsilon\right)-f^{\prime \prime}\left(t_{0}-\epsilon\right)=-2 \pi D \cos ^{2} \varphi .
$$

If the density of the Fisher zeros diverges as $\left|y-y_{0}\right|^{-\alpha}$ at the boundary of the Fisher area, the slopes of the cusps in $\operatorname{Re}\left\{f^{\prime}(t)\right\}$ inside the Fisher area diverge similarly. In the Haldane model this latter behavior occurs as shown in Fig. 3 .

These features are not specific to the Haldane model, but show up in a wide range of models, e.g., in the "half"-BHZ model [38], which is described by $\mathbf{d}_{\mathbf{k}}=$ $\left(A \sin k x, A \sin k y, \Delta+\cos k_{x}+\cos k_{y}\right)$, or in the lattice version of the chiral topological $p+i p$ superconductor with similar $\mathbf{d}_{\mathbf{k}}$ [39].

Conclusion. We have demonstrated the fundamental role of topology in quench dynamics. In the context of DPTs this means that if the topological number changes under the quench, DPTs have to occur, but the time evolution of the entanglement spectrum is also affected by the topology. We supported our findings with rigorous proofs for two-band models. An additional small interaction term does not destroy immediately the singularities in the dynamical free energy, but shifts them in time [19]. Correspondingly the Fisher zeros are only shifted and squeezed locally around the first few DPT points, which spoils the periodicity. Further away from the crossings, and for large interactions it is unknown how the Fisher structure is modified. Similarly, our preliminary numerical simulations indicate that DPTs are robust with respect to disorder. The general relation between topology and DPTs in systems with more (than two) bands and with interactions deserves further attention.

Previously the existence of DPTs was analyzed in comparison with equilibrium phase transitions $[11,14,15,18]$. Based on our results the topological nature of the equilibrium phase transition should be investigated as well. DPTs in two-dimensional systems are qualitatively different from those in one dimension as the cusps in the dynamical free energy appear in the first time derivative. The distinct behavior of the Fisher zeros together with different types of nonanalyticities in various systems may open a path to define universality classes in DPTs.

This research has been supported by the Hungarian Scientific Research Funds (Grants No. K101244, No. K105149, and No. K108676), by ERC Grant No. ERC-259374-Sylo, and by the Bolyai Program of the Hungarian Academy of Sciences.
[1] M. Z. Hasan and C. L. Kane, Rev. Mod. Phys. 82, 3045 (2010).

[2] X.-L. Qi and S.-C. Zhang, Rev. Mod. Phys. 83, 1057 (2011).
[3] A. Polkovnikov, K. Sengupta, A. Silva, and M. Vengalattore, Rev. Mod. Phys. 83, 863 (2011). 
[4] J. Dziarmaga, Adv. Phys. 59, 1063 (2010).

[5] G. Lamporesi, S. Donadello, S. Serafini, F. Dalfovo, and G. Ferrari, Nature Physics 9, 656 (2013).

[6] T. Kinoshita, T. Wenger, and D. S. Weiss, Nature (London) 440, 900 (2006).

[7] A. LeClair, G. Mussardo, H. Saleur, and S. Skorik, Nuclear Physics B 453, 581 (1995).

[8] M. Fagotti, arXiv:1308.0277.

[9] M. Campisi, P. Hänggi, and P. Talkner, Rev. Mod. Phys. 83, 771 (2011).

[10] T. B. Batalhão, A. M. Souza, L. Mazzola, R. Auccaise, R. S. Sarthour, I. S. Oliveira, J. Goold, G. De Chiara, M. Paternostro, and R. M. Serra, Phys. Rev. Lett. 113, 140601 (2014).

[11] M. Heyl, A. Polkovnikov, and S. Kehrein, Phys. Rev. Lett. 110, 135704 (2013).

[12] M. E. Fisher, in Boulder Lectures in Theoretical Physics (University of Colorado, Boulder, 1965), Vol. 453.

[13] T. Obuchi and K. Takahashi, Phys. Rev. E 86, 051125 (2012).

[14] F. Andraschko and J. Sirker, Phys. Rev. B 89, 125120 (2014).

[15] C. Karrasch and D. Schuricht, Phys. Rev. B 87, 195104 (2013).

[16] J. M. Hickey, S. Genway, and J. P. Garrahan, Phys. Rev. B 89, 054301 (2014).

[17] J. M. Hickey, arXiv:1403.5515.

[18] S. Vajna and B. Dóra, Phys. Rev. B 89, 161105 (2014).

[19] J. N. Kriel, C. Karrasch, and S. Kehrein, Phys. Rev. B 90, 125106 (2014).

[20] E. Canovi, P. Werner, and M. Eckstein, Phys. Rev. Lett. 113, 265702 (2014).

[21] M. Heyl, Phys. Rev. Lett. 113, 205701 (2014).

[22] M. Atala, M. Aidelsburger, J. T. Barreiro, D. Abanin, T. Kitagawa, E. Demler, and I. Bloch, Nature Physics 9, 795 (2013).
[23] G. Jotzu, M. Messer, R. Desbuquois, M. Lebrat, T. Uehlinger, D. Greif, and T. Esslinger, Nature (London) 515, 237 (2014).

[24] V. Mourik, K. Zuo, S. M. Frolov, S. R. Plissard, E. P. A. M. Bakkers, and L. P. Kouwenhoven, Science 336, 1003 (2012).

[25] S. Nadj-Perge, I. K. Drozdov, J. Li, H. Chen, S. Jeon, J. Seo, A. H. MacDonald, B. A. Bernevig, and A. Yazdani, Science 346, 602 (2014).

[26] S. Ryu, A. P. Schnyder, A. Furusaki, and A. W. W. Ludwig, New Journal of Physics 12, 065010 (2010).

[27] Y. Niu, S. B. Chung, C.-H. Hsu, I. Mandal, S. Raghu, and S. Chakravarty, Phys. Rev. B 85, 035110 (2012).

[28] M. P. L. Heyl, Nonequilibrium Phenomena in Many-Body Quantum Systems, Ph.D. thesis, Ludwig-Maximilians-Universität München, Munich, Germany, 2012.

[29] S. Ryu and Y. Hatsugai, Phys. Rev. Lett. 89, 077002 (2002).

[30] S. Mao, A. Yamakage, and Y. Kuramoto, Phys. Rev. B 84, 115413 (2011).

[31] X.-L. Qi, Y.-S. Wu, and S.-C. Zhang, Phys. Rev. B 74, 085308 (2006).

[32] M. Nakahara, Geometry, Topology and Physics (CRC Press, Boca Raton, 2003).

[33] M.-C. Chung, Y.-H. Jhu, P. Chen, and C.-Y. Mou, J. Phys.: Condens. Matter 25, 285601 (2013).

[34] M.-C. Chung, Y.-H. Jhu, P. Chen, C.-Y. Mou, and X. Wan, arXiv: 1401.0433.

[35] W. P. Su, J. R. Schrieffer, and A. J. Heeger, Phys. Rev. Lett. 42, 1698 (1979).

[36] F. D. M. Haldane, Phys. Rev. Lett. 61, 2015 (1988).

[37] K. Takahashi and T. Obuchi, J. Phys.: Conf. Series 473, 012023 (2013).

[38] B. A. Bernevig, T. L. Hughes, and S.-C. Zhang, Science 314, 1757 (2006).

[39] A. Bühler, N. Lang, C. V. Kraus, G. Möller, S. D. Huber, and H. P. Büchler, Nat. Commun. 5, 4504 (2014). 